\title{
Assemblies in Synthetic DNA (Nannochloropsis Species) Crown Cells with Bacillus subtilis
}

\section{Shoshi Inooka*}

Japan Association of Science Specialists, Japan

*Corresponding Author: Shoshi Inooka, Japan Association of Science Specialists, Japan.
Received: November 25, 2021

Published: December 13, 2021

(C) All rights are reserved by Shoshi Inooka.

\section{Abstract}

DNA crown cells are artificial cells in which the outer membrane is covered with DNA. DNA crown cells (artificial cells) can be easily synthesized using a sphingosine (Sph)-DNA-adenosine mixture (Synthetic DNA crown cells), and then cultured in egg white (DNA crown cells). Numerous varieties of both kinds of DNA crown cells have been prepared using a variety of different DNA sources.

To study the potential applications of DNA crown cells, the assemblies in synthetic DNA crown cells and B. subtilis that are formed when several kinds of synthetic DNA crown cells were mixed with B. subtilis were examined. In addition, the suppression of $B$. subtilis growth was observed. Further, target bacteria that were recovered from the assemblies were not $B$. subtilis (original bacteria), but Brevibacillus, which were produced by synthetic DNA crown cells which promoted the elongation of $B$. subtilis in the assembly. However, more information on the assembly and formation of DNA crown cells is required to better clarify the characteristics of these cells. The present experiment demonstrated for the first time that an assembly comprising synthetic DNA (Nannochloropsis) crown cells and B. subtilis was formed, that the assembly suppressed bacterial growth, and that bacterial transformation (i.e., from Bacillus to Brevibacillus was observed within the assembly. Moreover, cell-like objects were released from the assembly.

Keywords: Synthetic DNA; Nannochloropsis; Crown Cells; Assembly; Bacillus subtilis; Sphingoshine, Sphingoshine-DNA

\section{Introduction}

In 2012, the approaches for generating fully operational (i.e., self-replicating) artificial cells have been reported [1,2]. Such artificial cells are covered with DNA [3]. I named a DNA crown cell for such artificial cells [3]. These cells can be synthesized by mixing Sph-DNA and A-M compound-monolaurin (synthetic DNA crown cells), and can replicate when incubated in egg white (DNA crown cells) [9]. An unlimited number of both types of DNA crown cells can be theoretically prepared using these methods [4-7].

While it has been shown that self-replicating artificial cells (DNA crown cells) can be prepared with chemical agents, sphingo- sine, DNA, adenosine and monolaurin, it is not clear whether such cells can function in vivo.

As part of research into possible applications of DNA crown cells, it was previously demonstrated that synthetic DNA (Escherichia coli) crown cells have the ability to form an assembly with Bacillus subtilis [10].

It has also been shown that DNA crown cells can suppress bacterial growth, as well as induce cell death. In addition, DNA crown cells can also induce bacterial transformation [12] and bacterial elongation [13] within assemblies. Moreover, it has been suggested 
that new life, like DNA crown cells, may develop from such assemblies [11]. While it has been demonstrated that such assemblies can be formed using several kinds of synthetic DNA crown cells, more data on assembly formation is required. Hence, the present experiments examined whether assembly formation and growth suppression of bacteria can be achieved using synthetic DNA (Nannochloropsis) crown cells, which has not been tested to date.

\section{Materials and Methods}

Sphingoshine (Tokyo Kasei, Japan), DNA (extracted from Nannochloropsis species), adenosine (Sigma, USA and Wako, Japan), monolaurin (Tokyo Kasei, Japan), and a mixture of adenosine and monolaurin (AM) [9] were used in this study. Bacillus subtilis (natto) was purchased from Daikokuya, Nagoya, Japan. Dry B. subtilis was cultured at $37^{\circ} \mathrm{C}$ for $18 \mathrm{~h}$ on agar (Sanisupetsuku) growth medium (Azuwan, Japan). Then, bacteria were collected and inoculated into distilled water at a concentration of approximately $10^{6}$ cells $/ \mathrm{mL}$.

Synthetic DNA (Nannochloropsis) crown cells were prepared using Sph-DNA-AM, as described previously [6,8]. Briefly, $180 \mu \mathrm{g}$ of Sph $(10 \mathrm{mM})$ and $90 \mu \mathrm{L}$ of DNA $(1.7 \mu \mathrm{g} / \mu \mathrm{L})$ were mixed, and the mixture was then heated twice. Then, AM $(100 \mu \mathrm{L})$ was added and the mixture was incubated at $37^{\circ} \mathrm{C}$ for $15 \mathrm{~min}$. Then, an additional $30 \mu \mathrm{L}$ of monolaurin $(0.1 \mathrm{M})$ was added and the mixture was incubated at $37^{\circ} \mathrm{C}$ for $5 \mathrm{~min}$. This solution was used as a suspension for the synthetic DNA crown cells.

Fifty microliters of $B$. subtilis suspension was then added to 50 $\mu \mathrm{L}$ of synthetic DNA crown cells, the solution was mixed, and the mixture was incubated for $3 \mathrm{~h}$ at $37^{\circ} \mathrm{C}$. After removing an aliquot to observe the formation of the assemblies under a microscope, aliquots of $50 \mu \mathrm{L}$ were plated on agar and incubated at $37^{\circ} \mathrm{C}$ for $18 \mathrm{~h}$. After incubation, the number of colonies was counted and these colonies were used as a source of target bacteria. To identify the target bacteria, five colonies were randomly selected from the agar plate. Identification of target bacteria was performed using a matrix assisted laser desorption ionization (MALDI) Biotyper (Bruker, Germany). Briefly, proteins contained in the bacteria were identified by a MALDI Biotyper, and profiles were queried against the Compass Library database, which contains 2716 registered species.
Bacterial colonies identified as Brevibacillus were then cultured at $30^{\circ} \mathrm{C}$ for 2 days in Soybean Casein Digest (SCD) culture medium (BBL, Becton Dickinson, USA) followed by gram-staining with Favor G (Nissui Pharmaceutical, Japan). The appearance of the cultivated cells was then observed under a microscopy.

\section{Results and Discussion}

Microscopic observation of synthetic DNA (Nannochloropsis) crown cells

Figure 1 (arrow a) shows a typical synthetic DNA crown cell (round-shaped) measuring approximately $10 \mu \mathrm{m}$ in size. In addition, rod or fibrous-like synthetic DNA crown cell were also observed (Figure 1, arrow b). Arrow c in figure 1 shows a cluster of numerous fibrous synthetic DNA crown cells. Original bacteria $(B$. subtilis) were round or rod-shaped and ranged in size from approximately 3 to $6 \mu \mathrm{m}$ (picture not shown).

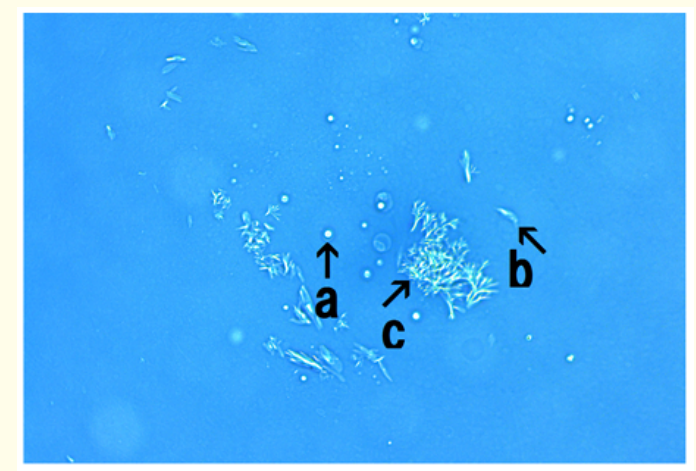

Figure 1: Microscopic appearance of synthetic DNA (Nannochloropsis) crown cells. A typical single DNA crown cell (arrow a) is round and measures approximately $10 \mu \mathrm{m}$ in diameter. Synthetic DNA crown cell-like fibrous cell (arrow b) and clusters containing synthetic fibrous-DNA crown cells (arrow c).

Formation of assemblies in mixed cultures of synthetic DNA (Nannochloropsis) crown cells with B. subtilis Bacillus subtilis were mixed with synthetic DNA crown cells and they were observed under a light microscope after incubation at $37^{\circ} \mathrm{C}$ for 60 minutes. An assembly, possibly degraded and divided in two parts, is shown in figure 2 (arrows a and b). Both parts contained numerous round 
cellular objects, but it was not clear whether they were DNA crown cells (Figure 2, arrows a and b). Figure 3 shows the microscopic appearance at 120 minutes after mixing cultures.

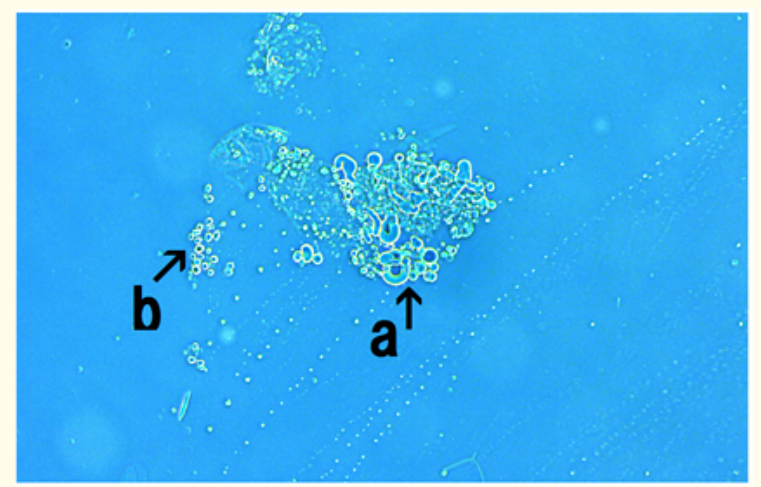

Figure 2: Assemblies obtained from mixtures of synthetic DNA (Nannochloropsis) crown cells with Bacillus subtilis after incubation at $37^{\circ} \mathrm{C}$ for 60 minutes. The assembly may degraded and divided in two parts (arrows a and b). Part a shows the appearance of many cell-like objects and their clusters. Part b shows a cluster to which several cell-like objects have bound.

After 120 minutes, no assemblies were observed within the sample tested, but many free objects of various shapes and sizes were observed (Figure 3, arrows a, b, c and d). One of these objects was cell-like (Figure 3 arrows a and b), while the others were objects that were shaped like fibers and long rods (Figure 3 arrows $c$ and d). In addition, a free bacterium was observed in figure 3 (arrow e). Figure 4 shows the assembly after 180 minutes of incubation at $37^{\circ} \mathrm{C}$; the assembly was similar in appearance to that observed after 60 minutes. A variety of round objects were observed together with the assembly (Figure 4 arrow), and it is clear, based on their structure, that these objects were not bacteria.

Growth suppression of bacteria by synthetic DNA (Nannochloropsis) crown cells

After culturing mixtures of DNA (Nannochloropsis) crown cells with B. subtilis for $3 \mathrm{~h}$ and incubating at $37^{\circ} \mathrm{C}, 50 \mu \mathrm{L}$ of the mixed culture was plated onto agar growing medium and the plates were incubated for $18 \mathrm{~h}$ at $37^{\circ} \mathrm{C}$. As shown in figure 5 (right), bacterial growth was observed on approximately half of the plates, whereas growth was observed over the entire plate in plates without DNA

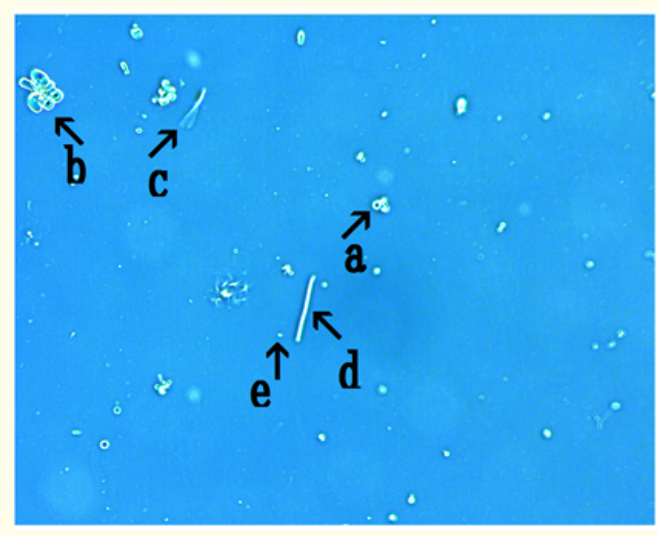

Figure 3: Microscopic appearance after incubation at $37^{\circ} \mathrm{C}$ for 120 minutes. Numerous bacteria-like objects of different sizes and shapes were observed in suspension. Round cell-like objects (arrow a). A cluster consisting of six round objects was observed (arrow b). Fibrous objects (arrow c) and fibers (arrow d) were observed. A free bacterium was observed (arrow e).

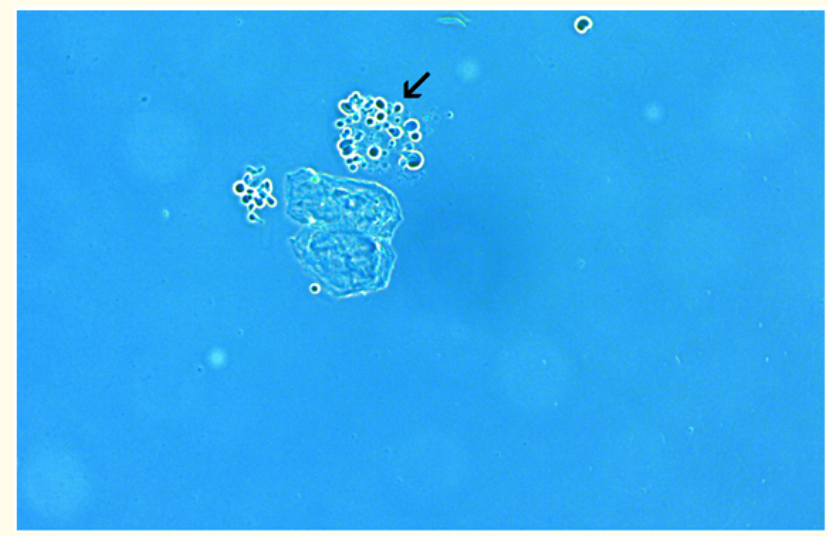

Figure 4: Microscopic appearance of assembly after incubation at $37^{\circ} \mathrm{C}$ for 180 minutes. A typical assembly after 180 minutes (arrow), showing a cluster of round cells (arrow). The assembly may consist of several cell-like objects.

crown cells (Figure 5, left). These results clearly showed that bacterial growth was inhibited in the mixed cell cultures, i.e., in cultures containing DNA (Nannochloropsis) crown cells and B. subtilis cells.

Identification of target cells based on results from the MALDI Biotyper 


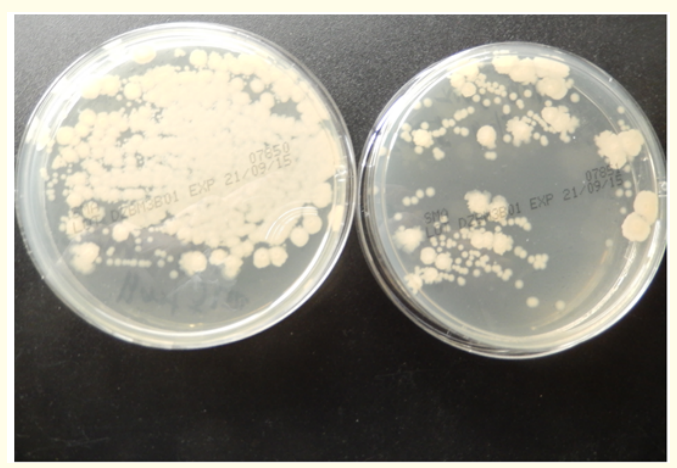

Figure 5: Effect of synthetic (Nannochloropsis) DNA crown cells on B. subtilis growth. After culturing synthetic DNA (Nannochloropsis) DNA crown cells together with $B$. subtilis for $3 \mathrm{~h}$ and incubating at $37^{\circ} \mathrm{C}$ bacterial growth was observed on half of the plate (right). Bacterial growth without cells (left).

A MALDI Biotyper was used to identify bacteria from five randomly selected colonies (Figure 5, right) and the samples were examined directly. The results showed that the target cells were not the original bacteria (i.e., B. subtilis), and that all of the bacteria from the five colonies were Brevibacillus borsteiensis.

These Brevibacillus cells could be identified in mixed cultures containing synthetic DNA (E. coli and Akoya pearl oyster) crown cells $[12,14]$ and Bacillus subtilis, but not in DNA (Human placenta) crown cells [11].

Gram-staining of bacteria identified as Brevibacillus borsteiensis

Next, a bacterial colony identified as Brevibacillus was cultivated on SCD and then stained with Favor G; the results are shown in figure 6. Bacteria were stained as pink rods, suggesting that they were gram-negative bacteria. Also, spores were not observed during cultivation for 48 hours. In general, Brevibacillus are gram-positive bacteria that form spores within cells. Therefore, the appearance of the stained bacteria did not correspond to the characteristics of known Brevibacillus.

Thus, the present experiments showed that assembly formation and growth suppression of bacteria were observed in the mixed cultures consisting of synthetic DNA (Nannochloropsis) crown cells and $B$. subtilis. In addition, these phenomena were also observed

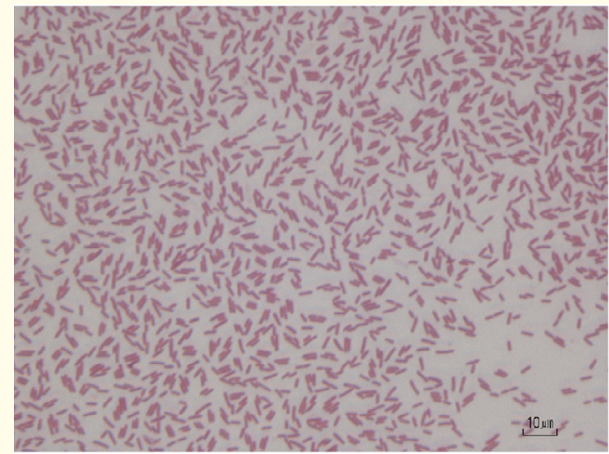

Figure 6: Gram-stain of Brevibacillus bacteria. Bacteria was separated from colonies shown in Figure 5 (right) and identified as a Brevibacillus by MALDI Biotyping. Bacteria were stained with pink-color. Scale bar: $10 \mu \mathrm{m}$.

for all combinations of the synthetic DNA crown cells that have been tested to date. However, it is possible that the appearance of assemblies may differ in shape and function. As described previously [10], the typical assemblies formed using synthetic DNA ( $E$. coli) crown cells are shown in figure 7 (arrow a).

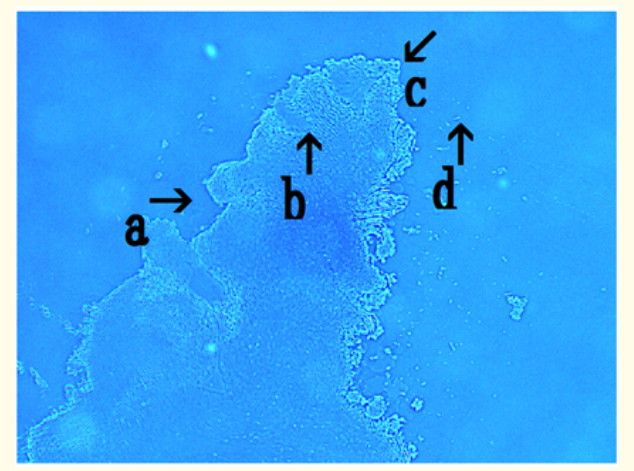

Figure 7: Assembly formed from mixed cultures of synthetic DNA (E. coli) crown cells with B. subtilis after 180 minutes. The assembly (arrow a) had a flat and firm appearance. Bacteria were observed within the assembly (arrow b). Cell-like objects were observed along the assembly (arrow c). A free bacterium was observed (arrow d).

Thus, its [10] appearance was flat and firm, whereas the assemblies obtained using synthetic DNA (Nannochloropsis) appeared 
pulpy and soft, as shown in figure 2. Bacteria could be observed within the assembly in synthetic DNA (E. coli) crown cells (Figure 7 arrow b) and cell-like objects were observed along the edges of assemblies (Figure 7 arrow c); however, no bacteria were observed within the assemblies of DNA (Nannochloropsis) crown cells, although cell-like objects were observed in suspension.

Elongated bacteria were observed in the assemblies formed using synthetic DNA (E. coli and human placenta) crown cells [13], but no elongated bacteria were observed using synthetic DNA (Nannochloropsis) crown cells. These findings show that there were clear differences among synthetic DNA crown cells.

In general, it was considered that assemblies were formed using components of the $B$. subtilis cell wall and synthetic DNA crown cells; for example, the ion binding apparatus in membrane components that both $B$. subtilis and synthetic DNA crown cells possess. Therefore, differences in the shape of assemblies may arise as a result of the characteristics of each type of synthetic DNA crown cell.

Also, the number of bacterial colonies that formed using mixed cultures of synthetic DNA (Nannochloropsis) crown cells occupied only half of the plate and it was shown that Nannochloropsis DNA crown cells were capable of suppressing bacterial growth. The capacity to suppress bacterial growth was evident in all of the synthetic DNA crown cells tested; however, it is possible that differences in the extent of this growth-suppression ability exists among synthetic DNA cells.

In a previous report [15], it was demonstrated that all synthetic DNA crown cells tested, including synthetic DNA (Nannochloropsis) crown cells (unpublished data), killed yeast. These findings suggest that there was no difference in this ability among synthetic DNA crown cells. These findings show that there may be a difference in the mechanisms that synthetic DNA crown cells employ to suppress the growth of $B$. subtilis and kill yeast, suggesting that synthetic DNA crown cells have at least two ways to kill or suppress bacteria.

On the other hand, objects similar to DNA crown cells were observed in assemblies of all synthetic DNA crown cells tested. In the present experiment using synthetic DNA (Nannochloropsis) crown cells, such objects were not observed to bind to the assembly and that they remained in suspension.
While it is clear that these objects were composed of synthetic DNA crown cells and their components, Sph or DNA, and B. subtilis, it is not clear whether they were living like DNA crown cells, which can multiply within egg white, or whether they were not capable of multiplication like liposomes.

In addition, bacteria could be isolated from colonies that survived being plated together with mixed cultures; these bacteria were identified as Brevibacillus based on the MALDI Biotype analysis. In addition, Brevibacillus was also found together with DNA crown cells (E. coli and Akoya pearl oyster) $[12,14]$.

The results clearly show that the original bacteria and target bacteria were different. However, gram-staining did not identify the target bacteria as being Brevibacillus borsteiensis. Brevibacillus are generally gram-positive bacteria, whereas the target bacteria were gram-negative. Since gram staining indicates the amount of peptidoglycan in the bacterial cell wall, with gram-positive cells having more peptidoglycan in the bacterial cell wall than gramnegative cells. Therefore, it is possible that the target bacteria may change the synthesis of peptidoglycan.

It is well kwon that Bacillus can alter their functions, such as during the transfer of genetic materials (including virus) from one bacterium to another and during conjugation among bacteria. Therefore, the findings of the present experiment imply that the genes of the original bacteria (Bacillus subtilis) changed within the assembly.

\section{Conclusion}

However, it is important to carefully consider whether there was an actual change in the genetic material within the assemblies of the bacteria including whether these are unique bacteria. Thus, in order to conclusively show that the present observations were based on genetic changes, further research may be needed on the underlying mechanisms associated with a change from Bacillus subtilis to Brevibacillus.

In summary, the present experiments were conducted to clarify whether synthetic DNA (Nannochloropsis) crown cells can form assemblies and suppress bacterial growth. The results showed that DNA crown cells form assemblies and suppress bacterial growth. Also, it is possible that bacterial transformation occurred within the assemblies and that new organism-like DNA crown cells were produced within the assemblies. 
The present studies are important in the life sciences, particularly on the origin of life and bacterial diversity, as well as in the field of biotechnology.

\section{Acknowledgements}

The author thanks Sho Morohoshi from Techno Suruga Laboratory Co., Ltd. for assistance with the MALDI Biotyper analysis and gram-staining.

\section{Bibliography}

1. Inooka S. "Preparation and cultivation of artificial cells". Applied Cell Biology 25, 13-18, 2012

2. Inooka S. "Preparation of Artificial Cells Using Eggs with Sphingosine-DNA". Journal of Chemical Engineering and Process Technology 17 (2016): 277.

3. Inooka S. "Aggregation of sphingosine-DNA and cell construction using components from egg white". Integrative Molecular Medicine 3.6 (2016): 1-5.

4. Inooka S. "Systematic Preparation of Bovine Meat DNA Crown Cells". Applied Cell Biology Japan 30 (2017): 13-16.

5. Inooka S. "Systematic Preparation of DNA (Akoya pearl oyster) Crown Cells". Applied Cell Biology Japan 31 (2018): 21-34.

6. Inooka S. "Preparation of DNA (Nannochloropsis species) Crown Cells Using eggs, sphingoshine and DNA, and subsequent cell recovery". Applied Cell Biology Japan 32 (2019): 5564.

7. Inooka S. "Preparation of Artificial Human Placental Cells". Applied Cell Biology 27 (2014): 43-49.

8. Inooka S. "DNA crown cells using sphingoshine, DNA and eggs-Preparation of DNA (Nannochloropsis) crown cells". In Y. Inamori, S. Inooka and T. Sakai (Eds), Oyo saibo shigen riyougaku. Taiyo Shobo Corporation (Niigata, Japan) 3 (2020): 91-120.

9. Inooka S. "Biotechnical and Systematic Preparation of Artificial Cells (DNA Crown Cells)". The Global Journal of Researches in Engineering 17.1 (2017).

10. Inooka S. "The assembly formation by co-cultures of DNA (Escherichia coli) crown cells with Bacillus subtilis cells". Journal of Biotechnology and Bioresearch 2.5 (2021).
11. Inooka S. "Microscopic observation of assemblies formed in mixtures of DNA (human placenta) crown cells with Bacillus subtilis". An Archive of Organic and In-organic Chemical Sciences 5.2 (2021): 658-664.

12. Inooka S. "Growth suppression of Bacillus subtilis in Co-cultures with DNA (Escherichia coli) Crown Cells, Including Possible Bacterial Transformation within the Assemblies". Bioscience and Biotechnology Journal 2.1 (2021): 22-25.

13. Inooka S. "Systematic DNA (Human Placenta) Crown Cells Elongate Bacillus subtills-Elongation Occurs with the Combination of Sphingosine and DNA". American Journal of Biomedical Science and Research 14.5 (2021): 419-424.

14. Inooka S. "Assembly and Bacterial Growth Suppression in the Co-cultures of Synthetic DNA (Akoya Pearl Oyster) Crown Cells with Bacillus subtilis". Applied Cell Biology Japan 34 (2021): 67-84.

15. Inooka S. "Synthetic DNA Crown Cells Kill Yeast: Relationship with Assembly Formation of Yeast". Civil Engineering Research Journal 12.2 (2021): CERJ.MS.ID555831.

\section{Assets from publication with us}

- Prompt Acknowledgement after receiving the article

- Thorough Double blinded peer review

- Rapid Publication

- Issue of Publication Certificate

- High visibility of your Published work

Website: www.actascientific.com/

Submit Article: www.actascientific.com/submission.php

Email us: editor@actascientific.com

Contact us: +919182824667 